\title{
Pickling Experimental Study on Preparation of Diesel Oil with Pyrolysis Oil from Waste Rubber
}

\author{
Wang Lei ${ }^{1,2,3}$, Wang Yun ${ }^{1}$, Jin Jie ${ }^{1,2}$ \\ 1, Department of Biological and Environmental Engineering, Hefei University; 2, Collaborative Invention \\ Center for Environmental Pollution Precaution and Restoration of Anhui, Hefei 230601, China;3, Anhui Key \\ Laboratory of Sewage Purification and Ecological Rehabilitation Materials
}

\begin{abstract}
The research has been done for removing asphaltene by pickling process of diesel oil from pyrolysis oil self-made by waste rubber in this paper, and the study showed that pickling effect of concentrated sulfuric acid was better than concentrated hydrochloric acid. The best pickling effect was found when the concentration of sulfuric acid was $18.4 \mathrm{~mol} / \mathrm{L}$, acid to oil ratio, namely, the amount of concentration of sulfuric acid to the amount of diesel oil ratio, was $25 \%$. This experiment proved that removing asphaltene by pickling process using concentrated sulfuric acid was remarkable.
\end{abstract}

Keywords: asphaltene, pyrolysis, diesel oil, pickling.

\section{INTRODUCTION}

With the rapid development of pyrolysis technology in recent years, the fate of waste rubber happened changed greatly. Seen from the relevant literature, the preparation of diesel oil using pyrolysis oil from waste rubber was feasible, and some characteristics of the diesel oil was better than 0\# diesel oil, which means that waste rubber have better utilization way[1,2,3,4].

However, the high level of asphaltene within the diesel oil can have an adverse impact on the diesel oil quality [2, 5, 6, 7]. Consequently pickling, an effective way to remove asphaltene, was crucial, and then the following factors need to be studied. Firstly, we need to confirm the dominant acid type. After by means of single factor experiments, the influencing factors like acid concentration, acid-to-oil ratio, stirring rate and immersion time were researched. Finally, we point out the optimum conditions of pickling process.

\section{MATERIALS AND EQUIPMENT \\ Materials}

Self-made pyrolysis oil (It came from waste rubber pyrolysis using High temperature pyrolysis furnace. Nitrogen gas as carrier gas, the flow of 50 $\mathrm{mL} / \mathrm{min}$; Pyrolysis temperature of 450 degrees centigrade for $10 \min [5,8,9,10,11]$.)

\section{Reagents}

Concentrated hydrochloric acid (AR); Concentrated sulfuric acid (AR); Sodium carbonate anhydrous (AR); Atlapulgite; Benzoic acid (AR).

\section{Equipment}

High temperature pyrolysis furnace (OTF-1200X); Universal furnace (DL-1); Circulating water type multipurpose vacuum pump (SHB-III); Blast dryer (DGX-9143B); Analytical balance (CP214); Six asynchronous automatic lifting agitator (JJ-6A); Constant temperature water bath oscillator (DKZ-2); Liquid gun (WD-2108); Microcomputer oxygen bomb calorimeter (XRY-1B).

Process

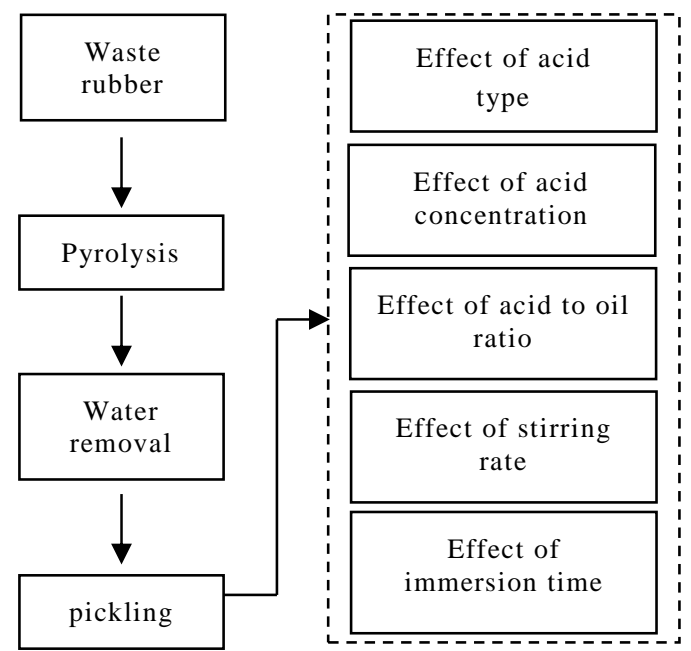

Fig.1. Pickling experiment flow graph 


\section{Methods}

The diesel oil sample was given calorific value test by microcomputer oxygen bomb calorimeter before and after the experiments in order to verify the effect of pickling [12, $13,14,15]$.

The experimental data was analyzed by using data fitting from SPSS (Statistical Product and Service Solutions) [14, 16, 17].

\section{Effects of acid type}

Figure 2 shows effect of asphaltene removal using two kinds of concentrated acid. A1 and A2, B1 and B2 are parallel sample of adding concentrated hydrochloric acid and adding concentrated sulfuric acid, respectively. It's not hard to see that under the same acid dosage, effect of asphaltene removal using concentrated sulfuric acid was superior to concentrated hydrochloric acid, and was twice concentrated hydrochloric acid. The color of diesel oil treated by concentrated sulfuric acid was brighter than concentrated hydrochloric acid (see figure 3, 4), for the asphaltene removal rate using concentrated sulfuric acid was higher than concentrated hydrochloric acid.

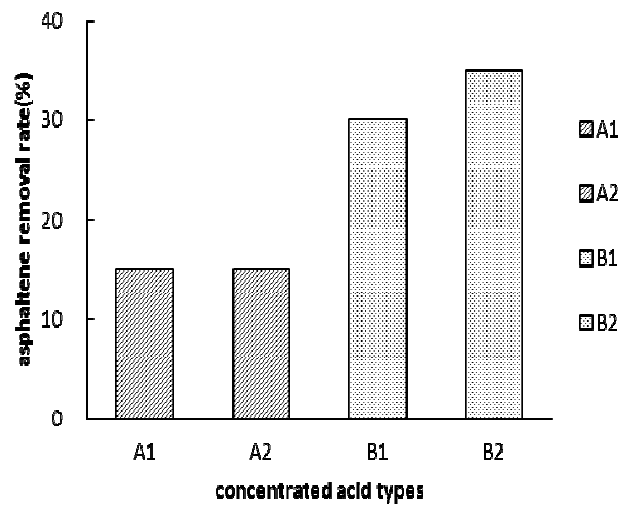

Fig.2. Effect of different concentrated acids on asphaltene removal

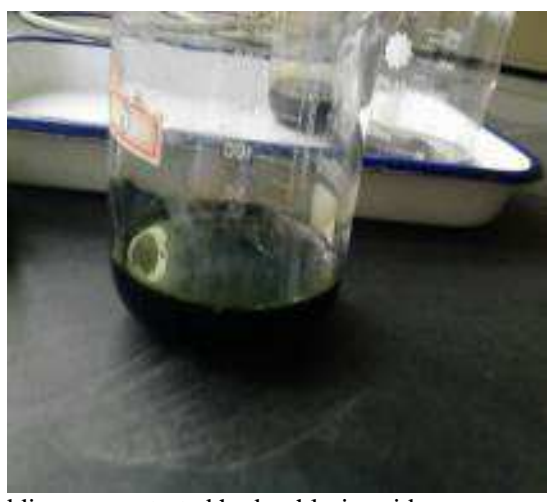

Fig.3. Adding concentrated hydrochloric acid

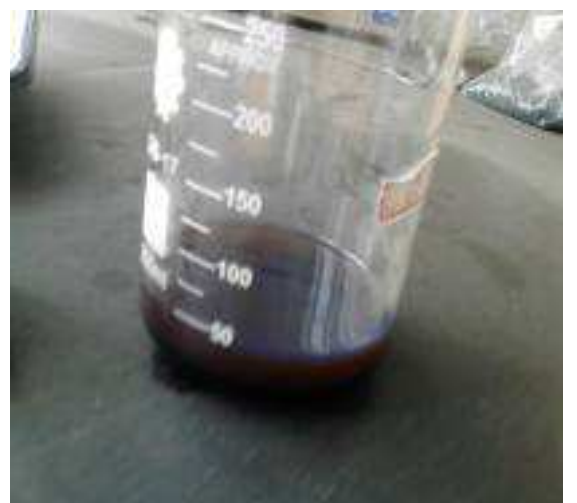

Fig. 4. Adding concentrated sulfuric acid

\section{Effect of acid concentration}

As shown in Table 1, asphaltene removal rate increases continuously with sulfuric acid concentration increases. It's worth mentioning that asphaltene removal rate has a sharp raise when the concentration of sulfuric acid was $18.4 \mathrm{~mol} / \mathrm{L}$. More importantly, Sulfuric acid with a concentration of $18.4 \mathrm{~mol} / \mathrm{L}$ not only realizes the highest asphaltenes removal rate, but also no additional increase of moisture content within diesel oil.

Table 1.

Effect of different sulfuric acid concentration on asphaltene removal

\begin{tabular}{cccccc}
\multicolumn{5}{c}{ sulfuric acid } \\
$\begin{array}{c}\text { concentration } \\
\text { (mol/L) }\end{array}$ & 1 & 2 & 5 & 10 & 18.4 \\
\hline $\begin{array}{c}\text { asphaltene removal } \\
\text { rate(\%) }\end{array}$ & 0.1 & 0.3 & 0.75 & 0.8 & 5 \\
\hline
\end{tabular}

\section{Effect of acid-to-oil ratio}

Asphaltene removal rate as shown in Table 2, effect of different amount of sulfuric acid to remove asphalt was different, but the removal efficiency was higher than $5 \%$. In addition, effect of asphaltene removal was the best when acid-to-oil radio of $25 \%$, the color of the diesel oil becomes bright, and the chroma of diesel oil was reduced to 5 degrees, close to the national standard.

Table 2.

Effect of different acid-to-oil ratio on asphaltene removal

\begin{tabular}{cccccc}
\hline $\begin{array}{c}\text { Sulfuric } \\
\text { acid-to-oil } \\
\text { ratio(\%) }\end{array}$ & 10 & 15 & 20 & 25 & 30 \\
\hline $\begin{array}{c}\text { asphaltene } \\
\text { removal rate(\%) }\end{array}$ & 7 & 10 & 6.5 & 30 & 5.3 \\
\hline
\end{tabular}

It can be seen from Figure 5 that the color of the middle sample was brighter than any others, the closest to the color of 0 \# diesel oil. 


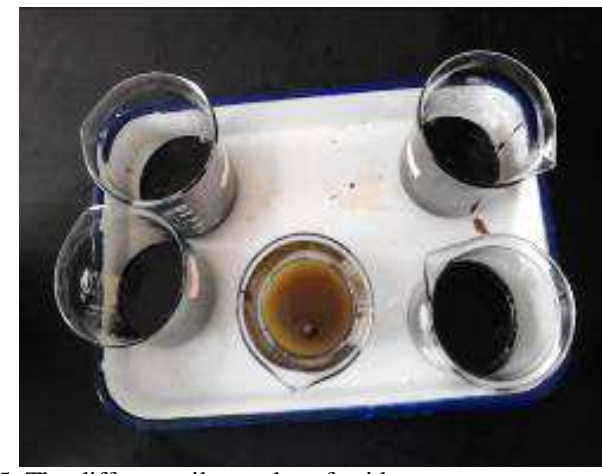

Fig.5. The different oil samples of acid

\section{Effect of stirring rate}

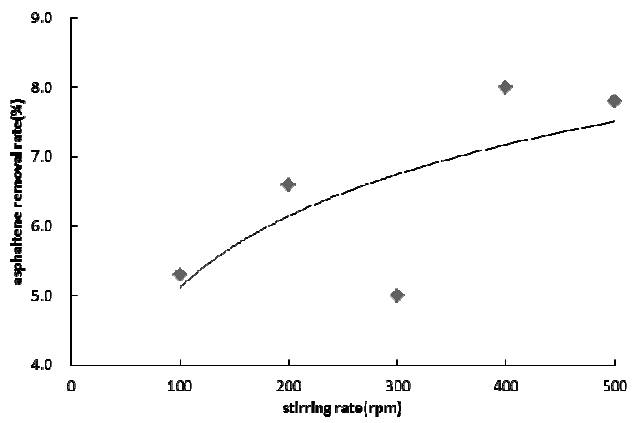

Fig.6. Effect of different rotational speeds on asphaltene removal

Asphaltene removal rate as shown in Figure 6, on the condition that the same concentration and amount of sulfuric acid, the amount of asphaltene removal increases along with the increasing of rotational speed. In the figure 6 , deviation tendency of the third point may be caused by improper operation. In addition, when the rotational speed increases to $400 \mathrm{rpm}$ or more, the amount of asphaltene removal increases slowly, even remain flat.

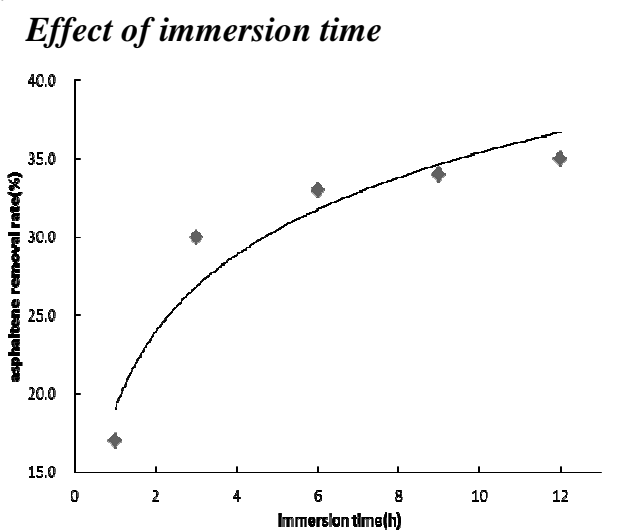

Fig.7. Effect of immersion time on asphaltene removal

With the immersion time increasing, effect of asphaltene removal was obvious. From Figuie 7 , when the immersion time was $0 \mathrm{~h}$ to $6 \mathrm{~h}$, effect of asphaltene removal was the most obvious; after $10 \mathrm{~h}$, the amount of asphaltene no longer increase, that was to say $10 \mathrm{~h}$ was the limited time of asphaltene removal.
Table 3 .

Calorific value of the diesel oil sample before and after the Pickling experiments

\begin{tabular}{lll}
\multicolumn{3}{c}{ Pickling experiments } \\
\hline Experimental condition & $\begin{array}{l}\text { After the } \\
\text { Experiment } \\
\text { Unit:kJ/kg }\end{array}$ & $\begin{array}{l}\text { Before the } \\
\text { Experiment } \\
\text { Unit:kJ/kg }\end{array}$ \\
\hline & & \\
Optimal acid concentration & 32067 & 27376 \\
& & \\
$\begin{array}{l}\text { Optimal acid-to-oil ratio } \\
\text { Optimal stirring rate }\end{array}$ & 36424 & \\
Optimal immersion time & 31157 & \\
\hline
\end{tabular}

As shown in Table 3, the calorific value of the diesel oil after pickling was generally higher than before pickling, and then the calorific value of $0 \#$ diesel oil was measured $40128 \mathrm{~kJ} / \mathrm{kg}$. Thus it can be seen that the effect of pickling was remarkable, the calorific value of diesel oil sample increased more than $30 \%$.

\section{CONCLUSIONS}

Effect of asphaltene removal using concentrated sulfuric acid was superior to concentrated hydrochloric acid.

During the pickling, the optimal concentration of sulfuric acid was $18.4 \mathrm{~mol} / \mathrm{L}$.

On the condition of acid-to-oil rate of $20 \%$, the chroma of diesel oil was reduced to 5 degrees.

The stirring rate of 200-400 rpm, effect of asphaltene removal was the best.

The optimal immersion time of asphaltene removal was $10 \mathrm{~h}$.

\section{REFERENCES}

[1] Jiang Yong, Liu Xiaolong, Liu Baoqing. Development of pyrolysis equipment for waste tires $[\mathrm{J}]$. Chemical industry exhibition.2002,21 (9): 681.

[2] Liu Tong. Study on pyrolysis of waste rubber: 5-6.

[3] Zhang Siwen. Utilization of waste tires in developed countries: Volume twenty-second, fourth, 8-9.

[4] Huang Li Ben, Gu Yusheng, Huang Jing. Powder rubber. Chemical Industry Press, 33-38.

[5] Han Fei, Li Zhikun. Recycling technology of waste tires in Japan, Beijing: 45.

[6] Kang Peiyu. Study on the treatment of industrial sludge by low energy plasma incineration technology. North China Municipal Engineering Design and Research Institute, Tianjin.

[7] Li Rulin. Current situation and development countermeasure of waste tire recycling industry in China, Beijing: 2003,3.

[8] The Ministry of Chemical Industry Ministry of personnel education, the Ministry of chemical industry education and training center. Renewable rubber production mechanism, process and quality inspection. Beijing: Chemical Industry Press.

[9] Sun Hongyou. Production of diesel oil by pyrolysis of waste oil [J], technology and market, 2001, 9.

[10] Guan Xiaoyan, Kong Fanzhong, Yang Jingfang, Zhang Wei. Heat treatment of metals in twenty-eighth volumes for $>2003$ twelfth, 50-53.

[11] Cheng Weimin. Waste oil refining diesel oil project design review. Tractors and agricultural vehicles > June 2012 thirty-ninth volume sixth. 
[12] Zhao Fenglan, Yan Jienian. Study on asphaltene inhibitors and clear agent. University of Petroleum (Beijing) EOR Research Center, University of Petroleum (Beijing) Institute of petroleum engineering. Oilfield chemistry, volume twenty-first, fourth, 311-312.

[13] Dong Liming, Yuen Wo Ping. Intensive land use [J]. China land science, 2000.

[14] Wang Yunqiang, Li Li, and. Research progress of biodiesel preparation technology $[\mathrm{J}]$. Anhui agricultural science, 2010
[15] Li Lilin, Du Wei, et al., et al. Preparation of biodiesel by lipase catalyzed by lipase in a new reaction medium [J]. Journal of process engineering, 2006 (in Chinese).

[16] Lv Dan, Du Wei, and. Study on the application of free lipase NS81006 in the preparation of biodiesel from oil containing acid $[\mathrm{J}]$. Chinese Journal of advanced chemical engineering, 2010.

[17] Wang Chang, Huang Xiaoming, et al. Development and application of biodiesel $[\mathrm{J}]$. Chemical industry and engineering, 2008 (in Chinese). 